Peter Luger*, Birger Dittrich, Stefan Mebs, Alexandra M.Z. Slawin and David A. Leigh

\title{
Analysis of two [2]catenanes based on electron densities from invariom refinement and results from DFT calculations
}

https://doi.org/10.1515/znb-2018-0179

Received August 24, 2018; accepted August 31, 2018

Abstract: Catenanes are of considerable interest as potential building blocks for molecular machines. The simplest [2]catenanes, Hopf links, consist of two macrocycles that are mechanically interlocked. This unusual architecture cannot be opened without breaking at least one covalent bond. Based on these structural characteristics, unusual properties on Hirshfeld or electrostatic potential surfaces could be expected. For a comparison of their structural and electronic properties, the electron densities (EDs) of two [2]catenanes, coded $\mathbf{H}_{2} \mathbf{2}$ and $\mathbf{H}_{4} \mathbf{L} \mathbf{7}$ in the original papers, were examined after application of the invariom formalism, relying on X-ray diffraction data collected earlier. The obtained electron density distributions were subjected to an analysis using the QTAIM formalism to yield bond and atomic properties. Moreover, molecular Hirshfeld surfaces and electrostatic potentials (ESP) were calculated. There are different types of intra- and intermolecular interactions in these two [2]catenanes. In addition to classical $\mathrm{N}-\mathrm{H} \cdots \mathrm{N}$ and $\mathrm{C}-\mathrm{H} \cdots \mathrm{O}$ hydrogen bonds, various types of $\pi \cdots \pi$ interactions in $\mathbf{H}_{2} \mathbf{2}$ and in $\mathbf{H}_{4} \mathbf{L} 7$ exist. Most of them are verified by local ED concentrations visible on the corresponding Hirshfeld surfaces, except for the parallel $\pi \cdots \pi$ interactions in $\mathbf{H}_{\mathbf{2}} \mathbf{2}$, which are either too weak or too diffuse to generate an ED signal on the Hirshfeld surface between the contributing aromatic rings. The electrostatic potentials (ESPs) were calculated and displayed on molecular surfaces. The interaction in the

*Corresponding author: Peter Luger, Institut für Chemie und Biochemie, Anorganische Chemie, Freie Universität Berlin, Fabeckstraße 36a, Berlin D-14195, Germany, e-mail: lugerp@zedat. fu-berlin.de

Birger Dittrich: Institut für Anorganische Chemie und Strukturchemie, Heinrich-Heine-Universität Düsseldorf, Universitätsstraße 1, Düsseldorf D-40225, Germany

Stefan Mebs: Institut für Experimentalphysik, Freie Universität Berlin, Arnimalle 14, Berlin D-14195, Germany

Alexandra M.Z. Slawin: School of Chemistry, University of St. Andrews, Purdie Building, St. Andrews, Fife KY16 9ST, UK

David A. Leigh: School of Chemistry, The University of Manchester, Oxford Road, Manchester M13 9PL, UK cavity of one macrocycle with the penetrated fragment of the second one was examined and it was found that corresponding to the above-mentioned contacts attractive and repulsive interactions exist. Additionally the ED was examined using results of density functional calculations, including non-covalent interaction index (NCI) and electron localizability indicator (ELI-D) surface analysis, complementing experimental findings.

Keywords: catenanes; DFT calculations; electron density; invariom formalism; molecular machines.

\section{Introduction}

Catenanes and rotaxanes are of increasing interest as potential building blocks for molecular machines [1] (and references cited therein). In their simplest forms, [2]catenanes consist of two macrocycles that are mechanically interlocked (Hopf links). This unusual structural architecture cannot be opened without breaking at least one covalent bond. This non-covalent interaction of the two macrocycles allows a high mutual mobility of the contributing molecules, which can be exploited in the design of larger mobile systems [2].

The current interest in catenane structural properties is also supported by the fact that more than 300 catenane $\mathrm{X}$-ray structures are listed in the Cambridge structural data base (CSD) [3]. Interestingly, if the conditions " $R<0.075$ " and "no disorder" are added to the corresponding query, the number of entries reduces to less than 30 . This is obviously an indication of the high mobility of the contributing macrocycles.

As far as we know, an electron density (ED) study in this class of compounds has not been reported, although based on the unique structural characteristics unusual properties, for example on Hirshfeld or electrostatic potential surfaces would make such study interesting. Even in the case of the simple [2]catenanes the structures consist normally of more than 100-150 atoms which would make an ED study on high resolution X-ray data a challenge. However, the invariom formalism, which was introduced several years before [4, 5], allows electron 
density work on conventional low resolution X-ray data sets, because the aspherical description of the atoms is entered from a library of fixed multipole contributions to the refinement. Hence, also larger structures can be examined with moderate effort.

We carried out invariom based ED studies on the data sets of the following X-ray structures:

1. The [2]catenane coded $\mathbf{H}_{2} \mathbf{2}$ in Ref. [6], CSD code LASVIL;

2. The [2]catenane coded $\mathbf{H}_{4} \mathbf{L} \mathbf{7}$ in Ref. [7], CSD code BOXCUN, which crystallizes with two molecules acetonitrile.

Both X-ray structures were derived from very accurate data sets, measured at $93 \mathrm{~K}$ and resolutions of $(\sin \theta / \lambda)_{\max }=0.60 \AA^{-1}$. Both are free of disorder, a favorable (but not essential) provision for invariom application. We believe them the most suited data sets in the CSD for invariom studies in the class of catenanes. This study was complemented by results from density functional theory.

\section{Results and discussion}

\subsection{Discussion of the invariom results}

The asymmetric units of the crystal structures are shown in Fig. 1 in ORTEP representations [8, 9], both macrocycles are displayed separately. The atomic numbering scheme
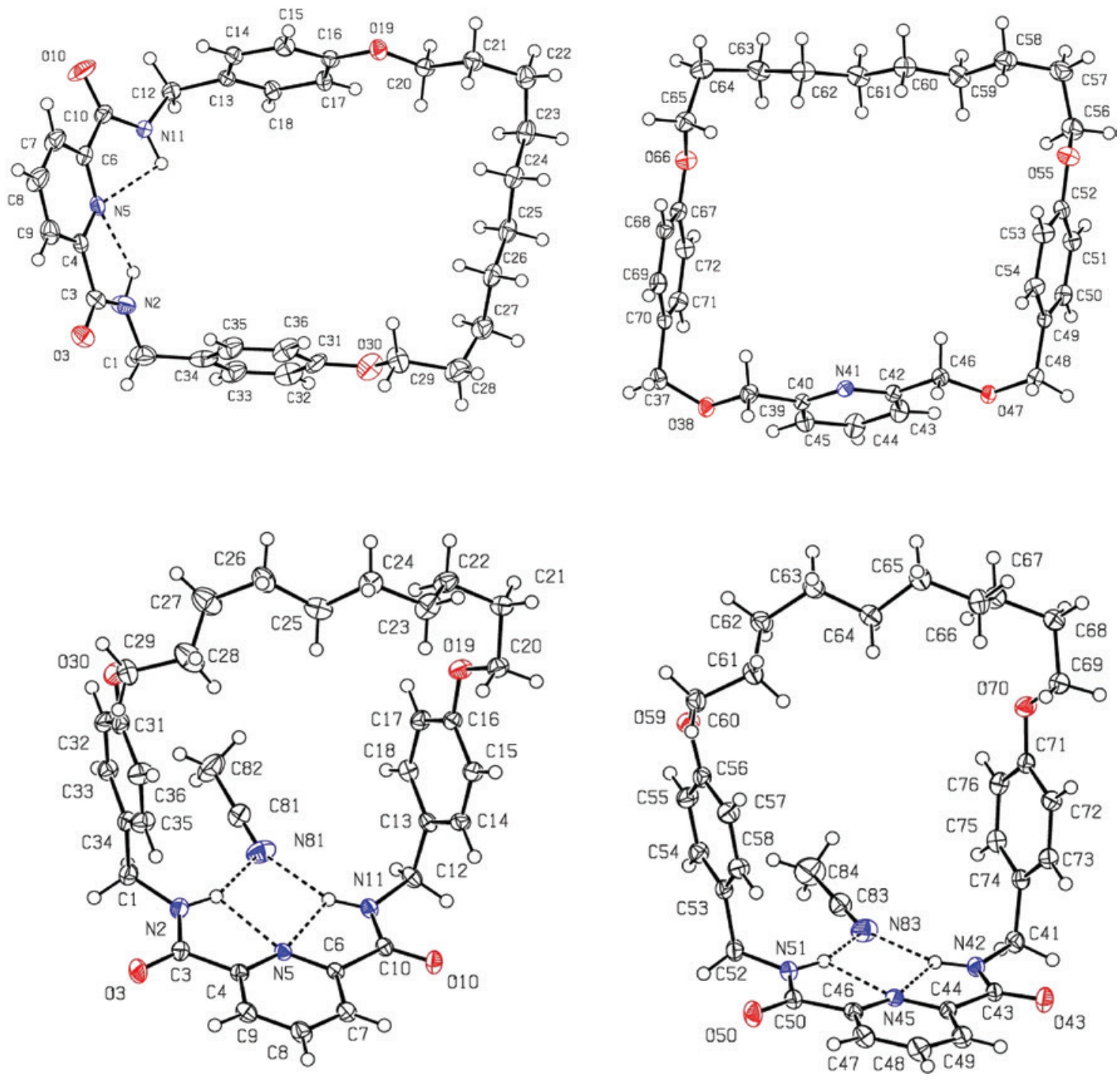

Fig. 1: Above: ORTEP representations [8,9] of the two contributing molecules of $\mathbf{H}_{2} \mathbf{2}$, atom numbering as in Ref. [6], macrocycle left, thread right [6]. Below: ORTEP representations of the two contributing molecules of $\mathbf{H}_{4}$ L7, atom numbering as in Ref. [7]. Mol. 1 left, mol. 2 right. 
taken from Ref. [6] and [7] was kept. We note that the two contributing molecules of $\mathbf{H}_{\mathbf{2}} \mathbf{2}$ are chemically different, designated as macrocycle and thread in Ref. [6]. In $\mathbf{H}_{4} \mathbf{L} 7$ the two contributing macrocycles are chemically identical and equal to the macrocycle of $\mathbf{H}_{\mathbf{2}} \mathbf{2}$. Two acetonitrile molecules complete the crystal structure of $\mathbf{H}_{4} \mathbf{L} 7$ as solvate molecuels. Nothing has to be discussed with respect to the bonding geometry. Bond lengths, for example, do not differ significantly from the ones reported in Ref. [6] and [7].

For $\mathbf{H}_{2} \mathbf{2}$ the $\mathrm{N}-\mathrm{H}$ hydrogen atoms are donors of three hydrogen bonds (HBs), see Figs. 1 and 2, and Table 1. Two of them are intramolecular within the macrocycle with
$\mathrm{N}(5)$ of the pyridine ring as a common acceptor. The third one $(\mathrm{N}(11)-\mathrm{H}(11 \mathrm{~N}) \cdots \mathrm{N}(41))$ is intermolecular having the pyridine nitrogen atom of the thread as acceptor. As listed in Table 1 three close $\mathrm{C}-\mathrm{H} \cdots \mathrm{O}$ contacts exist to carbonyl oxygen atoms of symmetry related catenanes. The intermolecular interactions are reflected by the Hirshfeld surfaces [11, 12] displayed in Fig. 3 [13]. When the aspherical ED is mapped by a color code onto this surface, ED concentrations are emphasized so that sites and strengths of intermolecular interactions become visible (see Fig. 3). Consequently, the color is most pronounced above the hydrogen atom $\mathrm{H}(11)$ which is the donor of the HB between the macrocycle and the thread situated
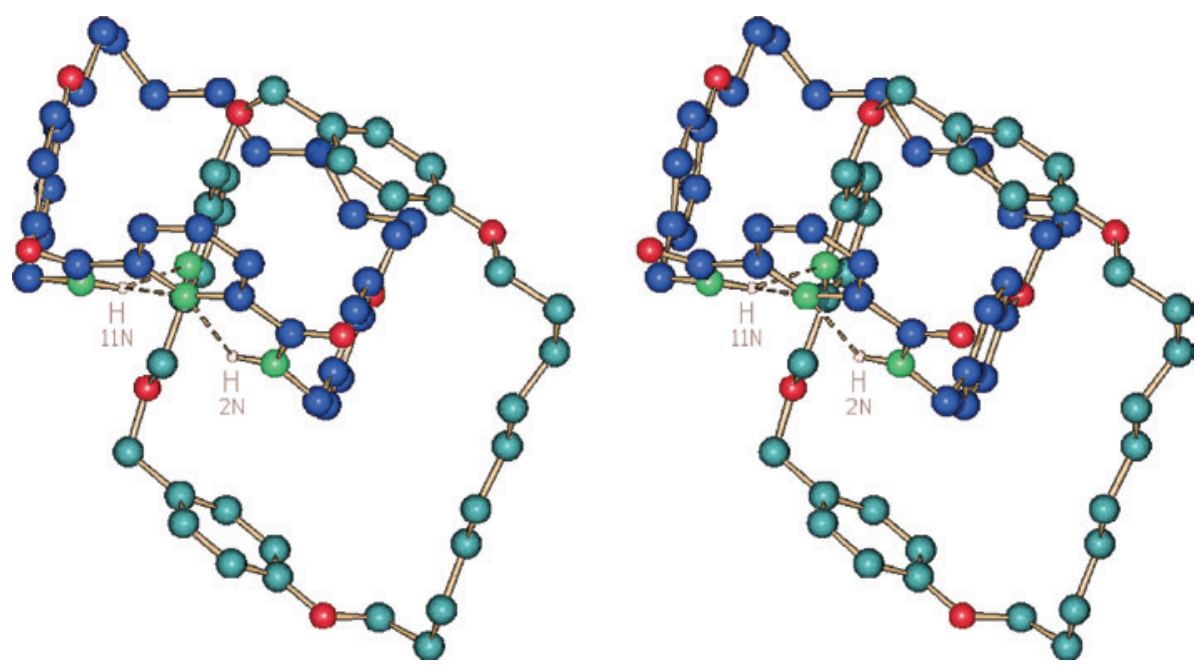

Fig. 2: Stereo representation of the complete [2]catenane $\mathbf{H}_{2}$ 2, generated with SCHAKAL [10]. Carbon atoms drawn in blue belong to the macrocycle, the ones of the thread are in turquoise. For clarity only hydrogen atoms contributing to $\mathrm{N}-\mathrm{H} \cdots \mathrm{N}$ hydrogen bonds are drawn. The corresponding representation for $\mathbf{H}_{4} \mathbf{L 7}$ is shown in the Supporting Information available online (Fig. S1).

Table 1: Summary of hydrogen bonding properties, data from PLATON [9] and XDProP [14].

\begin{tabular}{|c|c|c|c|c|c|c|c|}
\hline Model & D-H $\cdots A$ & $D \cdots A(\AA)$ & $H \cdots A(\AA)$ & $D-H \cdots A($ deg $)$ & $\rho\left(\mathrm{r}_{\mathrm{BCP}}\right)\left(e \AA^{-3}\right)$ & $\nabla^{2} \rho\left(\mathrm{r}_{\mathrm{BC}}\right)\left(e \AA^{-5}\right)$ & Type \\
\hline $\mathrm{H}_{2} 2$ & $N(2)-H(2 N) \cdots N(5)$ & $2.641(2)$ & $2.18(2)$ & $107.0(1)$ & & & Mol1/Mol1 \\
\hline $\mathrm{H}_{2} 2$ & $N(11)-H(11 N) \cdots N(5)$ & $2.712(2)$ & $2.33(2)$ & 102.2(1) & & & Mol1/Mol1 \\
\hline $\mathrm{H}_{2} 2$ & $N(11)-H(11 N) \cdots N(41)$ & $3.145(2)$ & $2.28(2)$ & $146.5(1)$ & 0.08 & 1.28 & Mol1/Mol2 \\
\hline $\mathrm{H}_{2} 2$ & $\mathrm{C}(22)-\mathrm{H}(22 \mathrm{~B}) \cdots \mathrm{O}(3)$ & $3.475(2)$ & $2.50(2)$ & $166.5(1)$ & 0.05 & 0.71 & Mol1/Mol1 ${ }^{\mathrm{a}}$ \\
\hline $\mathrm{H}_{2} 2$ & $\mathrm{C}(48)-\mathrm{H}(48 \mathrm{~A}) \cdots \mathrm{O}(3)$ & $3.418(2)$ & $2.51(2)$ & $152.4(1)$ & 0.05 & 0.74 & Mol2/Mol1 ${ }^{\mathrm{b}}$ \\
\hline $\mathrm{H}_{2} 2$ & $\mathrm{C}(54)-\mathrm{H}(54 \mathrm{~A}) \cdots \mathrm{O}(10)$ & $3.277(2)$ & $2.42(2)$ & $150.0(1)$ & 0.06 & 0.89 & Mol2/Mol1 ${ }^{\mathrm{c}}$ \\
\hline $\mathrm{H}_{4} \mathrm{L7}$ & $N(2)-H(2 N) \cdots N(5)$ & $2.684(2)$ & $2.21(2)$ & 108.3(1) & & & Mol1/Mol1 \\
\hline $\mathrm{H}_{4} \mathrm{L7}$ & $N(11)-H(11 N) \cdots N(5)$ & $2.685(2)$ & $2.25(2)$ & $105.8(1)$ & & & Mol1/Mol1 \\
\hline $\mathrm{H}_{4}^{4} \mathrm{L7}$ & $N(42)-H(42 N) \cdots N(45)$ & $2.682(2)$ & $2.22(2)$ & 107.2(1) & & & Mol2/Mol2 \\
\hline $\mathrm{H}_{4} \mathrm{L7}$ & $N(51)-H(51 N) \cdots N(45)$ & $2.674(2)$ & $2.22(2)$ & 106.9(1) & & & Mol2/Mol2 \\
\hline $\mathrm{H}_{4} \mathrm{L7}$ & $\mathrm{N}(2)-\mathrm{H}(2 \mathrm{~N}) \cdots \mathrm{N}(81)$ & $3.234(2)$ & $2.40(2)$ & 143.2(1) & 0.06 & 0.91 & Mol1/MeCN1 \\
\hline $\mathrm{H}_{4} \mathrm{L7}$ & $N(11)-H(11 N) \cdots N(81)$ & $3.141(2)$ & $2.28(2)$ & 146.7(1) & 0.08 & 1.21 & Mol1/MeCN1 \\
\hline $\mathrm{H}_{4} \mathrm{L7}$ & $N(42)-H(42 N) \cdots N(83)$ & $3.120(2)$ & $2.26(2)$ & $145.6(1)$ & 0.08 & 1.27 & Mol2/MeCN2 \\
\hline $\mathrm{H}_{4} \mathrm{L7}$ & $N(51)-H(51 N) \cdots N(83)$ & $3.170(2)$ & $2.31(2)$ & $145.8(1)$ & 0.07 & 1.08 & Mol2/MeCN2 \\
\hline $\mathrm{H}_{4}^{4} \mathrm{L7}$ & $C(33)-H(33 A) \cdots O(10)$ & $3.345(2)$ & $2.40(2)$ & $176.0(1)$ & 0.06 & 0.90 & Mol1/Mol1 ${ }^{\mathrm{d}}$ \\
\hline $\mathrm{H}_{4}^{4} \mathrm{L7}$ & $C(54)-H(54 A) \cdots O(43)$ & $3.509(2)$ & $2.56(2)$ & $174.6(1)$ & 0.04 & 0.62 & $\mathrm{Mol} 2 / \mathrm{Mol}^{\mathrm{d}}$ \\
\hline
\end{tabular}

aSymmetry code: $-1+x, 1+y, z$; ${ }^{\mathrm{b}}$ symmetry code: $-1+x, y, z$; 'symmetry code: $1-x, 1-y ; 1-z$; ${ }^{\mathrm{d}}$ symmetry code: $1+x, y, z$. 

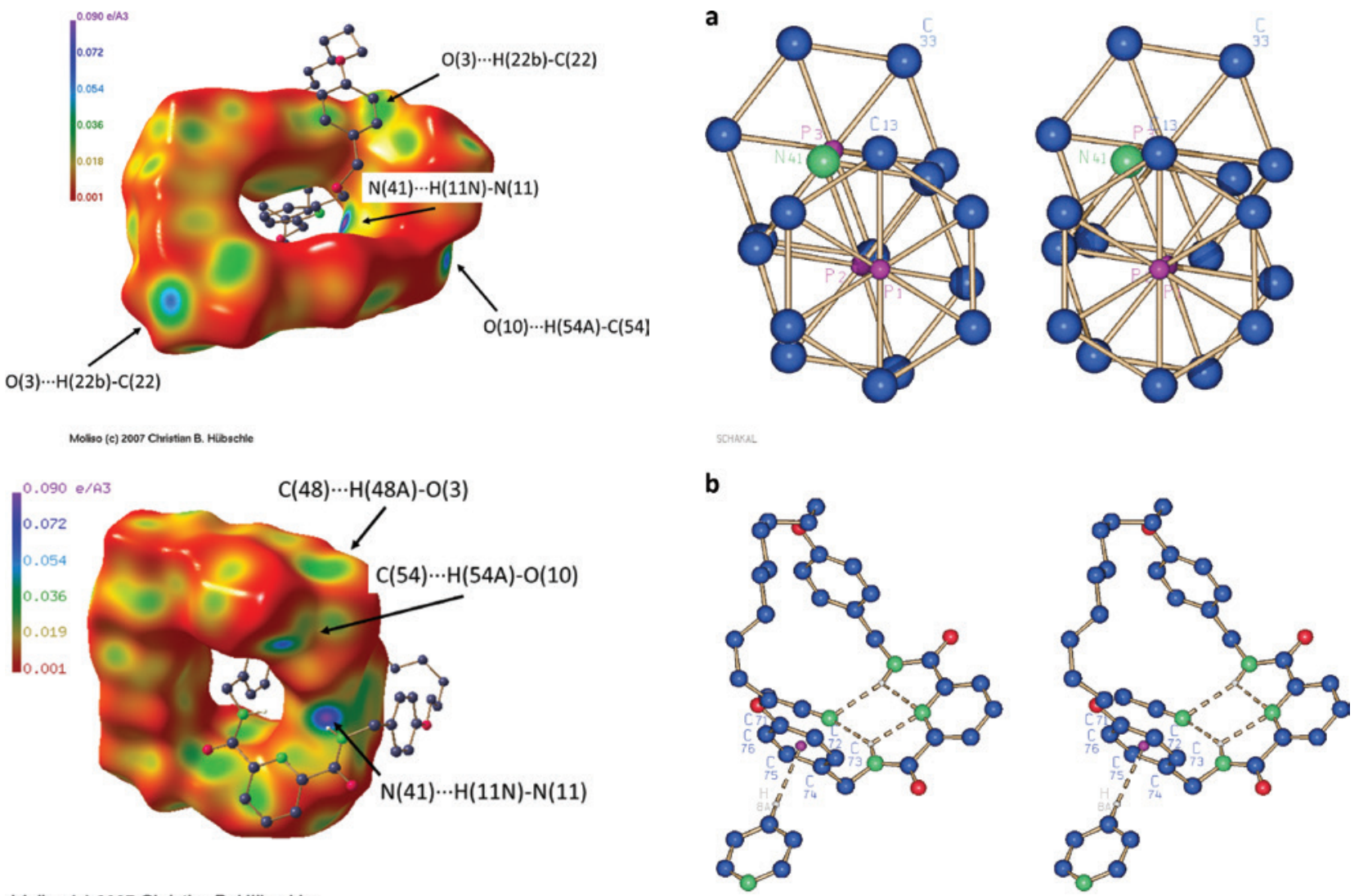

Moliso (c) 2007 Christian B. Hübschle

Fig. 3: Top: Hirshfeld surfaces of the macrocycle of $\mathbf{H}_{2} \mathbf{2}$, the thread is shown as ball-and-stick representation; bottom: thread Hirshfeld surface, the macrocycle is shown as ball-and-stick representation. For clarity, in both ball-and-stick representations the hydrogen atoms are omitted, representation with MoLIso [13].

in the cavity of the macrocycle. It is the only HB between macrocycle and thread. The two intramolecular $\mathrm{N}-\mathrm{H} \cdots \mathrm{N}$ HBs are not displayed on the Hirshfeld surface. As listed in Table 1 three close $\mathrm{C}-\mathrm{H} \cdots$ O contacts exist to the carbonyl oxygen atoms of symmetry related catenanes. They also appear on the Hirshfeld surfaces of either the macrocycle or the thread as strong ED concentrations as indicated by the color code, so that the two Hirshfeld surfaces provide a general impression about sites and strengths of intermolecular interactions.

It is worth to mention that the pyridine ring $\mathrm{C}(40)-$ $\mathrm{N}(41) \cdots \mathrm{C}(45)$ of the thread in the interior of the macrocyclic cavity is parallel to the phenyl rings $C(13) \cdots C(18)$ and $\mathrm{C}(31) \cdots \mathrm{C}(36)$ of the macrocycle with interplanar distances and angles of 3.7/3.8 $\AA$ and 9.4/2.9 ${ }^{\circ}$, respectively, indicating two types of $\pi \cdots \pi$ interactions. As illustrated in Fig. 4, the pyridine nitrogen atom is located above the center of the phenyl ring $\mathrm{C}(31) \cdots \mathrm{C}(36)$ indicating a "parallel displaced" arrangement, whereas the pyridine ring and the phenyl ring $\mathrm{C}(13) \cdots \mathrm{C}(18)$ are in the energetically

Fig. 4: Illustration of the $\pi \cdots \pi$ interactions. (a) $\mathbf{H}_{2}$ 2: phenyl rings $C(13) \cdots C(18), C(31) \cdots C(36)$ and the pyridine ring $C(40)-$ $\mathrm{N}(41) \cdots \mathrm{C}(45)$ are parallel. The pyridine nitrogen atom sits above the center of phenyl ring $C(31) \cdots C(36)$ indicating a "parallel displaced" arrangement; the pyridine ring and the phenyl ring $\mathrm{C}(13) \cdots \mathrm{C}$ (18) are in the energetically less favorable "sandwich" arrangement. (b) $\mathrm{H}_{4}$ L7: $\mathrm{C}(8)-\mathrm{H}(8 \mathrm{~A})$ of the pyridine ring in molecule 1 approaches the center of the phenyl ring $C(71) \cdots C(76)$ of molecule 2 (related via an inversion center) in the sense of a T-shaped edge-to-face $\pi \cdots \pi$ interaction; $\mathrm{H} \cdots \pi$ distance $2.56 \AA$. Centers of the aromatic rings in (a) and (b) are shown in magenta. Stereo representations are drawn with SchaKal [10].

less favorable "sandwich" arrangement. In the cavity of the thread the parallel displaced $\pi \cdots \pi$ interaction between pyridine ring $\mathrm{C}(40)-\mathrm{N}(41) \cdots \mathrm{C}(45)$ and phenyl ring $\mathrm{C}(31) \cdots \mathrm{C}(36)$ still exists. This phenyl ring sees on its opposite face the aliphatic chain $C(58) \cdots C(63)$.

The two pyridine rings of macrocycle and thread are almost perpendicular to each other, as already mentioned in Ref. [6].

For $\mathbf{H}_{4} \mathbf{L} \mathbf{7}$ the intra- and intermolecular contact pattern is very comparable for the two interlocked molecules. The $\mathrm{N}-\mathrm{H}$ groups are donors of bifurcated HBs to the accepting nitrogen atom of the pyridine ring (intramolecular) and to the nitrogen atom of a MeCN molecule 
(intermolecular). As for $\mathbf{H}_{2} \mathbf{2}$, attractive $\mathrm{C}-\mathrm{H} \cdots \mathrm{O}$ contacts exist (Table 1).

A close inspection of the $\mathbf{H}_{\mathbf{4}} \mathbf{L} \mathbf{7}$ Hirshfeld surfaces makes a further type of interaction with a rather strong ED concentration visible, which is not easy to recognize from distance considerations only. It is from $\mathrm{C}(8)-\mathrm{H}(8 \mathrm{~A})$, hence from the para $\mathrm{C}-\mathrm{H}$ of the pyridine ring in molecule 1 , to the center of the phenyl ring $\mathrm{C}(71) \cdots \mathrm{C}(76)$ of molecule 2 (related via an inversion center) in the sense of a T-shaped edge-to-face $\pi \cdots \pi$ interaction with a $\mathrm{H} \cdots \pi$ distance of $2.56 \AA$ (see Fig. 4b). This contact is expressed by a pronounced ED concentration above $\mathrm{H}(8 \mathrm{~A})$ (see Fig. 5, below) and in a bowl shaped cavity at the site of the accepting phenyl ring visible on the Hirshfeld surface of molecule 2 (Fig. 5, above).

Within one macrocycle, the phenyl rings are almost parallel to each other and perpendicular to the pyridine ring. In contrast to $\mathbf{H}_{\mathbf{2}} \mathbf{2}$ the pyridine rings are parallel to each other (not perpendicular) and a parallel arrangement
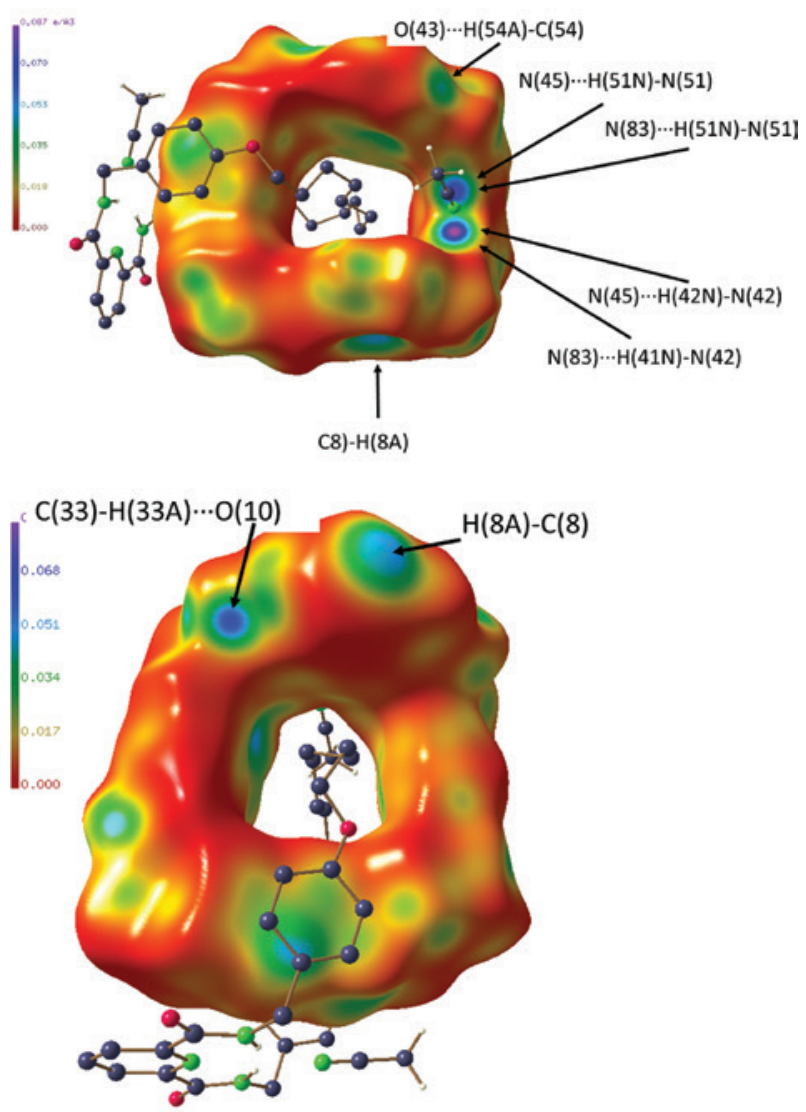

Moliso (c) 2007 Christian B. Hübschle

Fig. 5: Top: Hirshfeld surface of molecule 2 of $\mathbf{H}_{4}$ L7; molecule 1 is shown as ball-and-stick representation. Bottom: Hirshfeld surface of molecule 1; molecule 2 is shown as ball-and-stick representation. For clarity, in both ball-and-stick representations the hydrogen atoms are omitted, representation with MoLıso [13]. of three aromatic rings to yield $\pi \cdots \pi$ interactions as in $\mathbf{H}_{2} \mathbf{2}$ does not exist in $\mathbf{H}_{4} \mathbf{L} \mathbf{7}$.

In total, it follows that the Hirshfeld surfaces shown in Figs. 3 and 5 display the different types of interactions in the two catenanes, except for the parallel $\pi \cdots \pi$ interactions in $\mathbf{H}_{2} \mathbf{2}$, which are either too weak or too diffuse to generate an electron density signal on the Hirshfeld surface between the contributing aromatic rings. In contrast, the T-shaped edge-to-face interaction in $\mathbf{H}_{4} \mathbf{L} 7$ is strong enough to generate ED concentrations on both the donor and the acceptor site.

To illustrate the aspherical deformation electron density qualitatively, the static deformation density in the plane $\mathrm{N}(2), \mathrm{N}(11), \mathrm{N}(81)$ is represented for molecule 1 of $\mathbf{H}_{4} \mathbf{L} 7$ in Fig. 6. It is the plane of the bifurcated $\mathrm{N}-\mathrm{H} \cdots \mathrm{N}$ hydrogen bonds, where the donor N-H's are shown to be orientated towards the accepting lone-pair regions of the nitrogen atom.

For a quantitative analysis Bader's QTAIM formalism [15] was applied to yield bond critical points (BCPs) and atomic properties. For a summary of BCP properties see Supporting Information, Table S1.

Atomic properties were calculated by integration over the atomic basins bound by zero flux surfaces of the gradient vector field $\nabla \rho(\mathbf{r})$, which subdivide a structure into transferable substructures. The algorithm introduced by Volkov et al. [16] as implemented in the XDPRoP

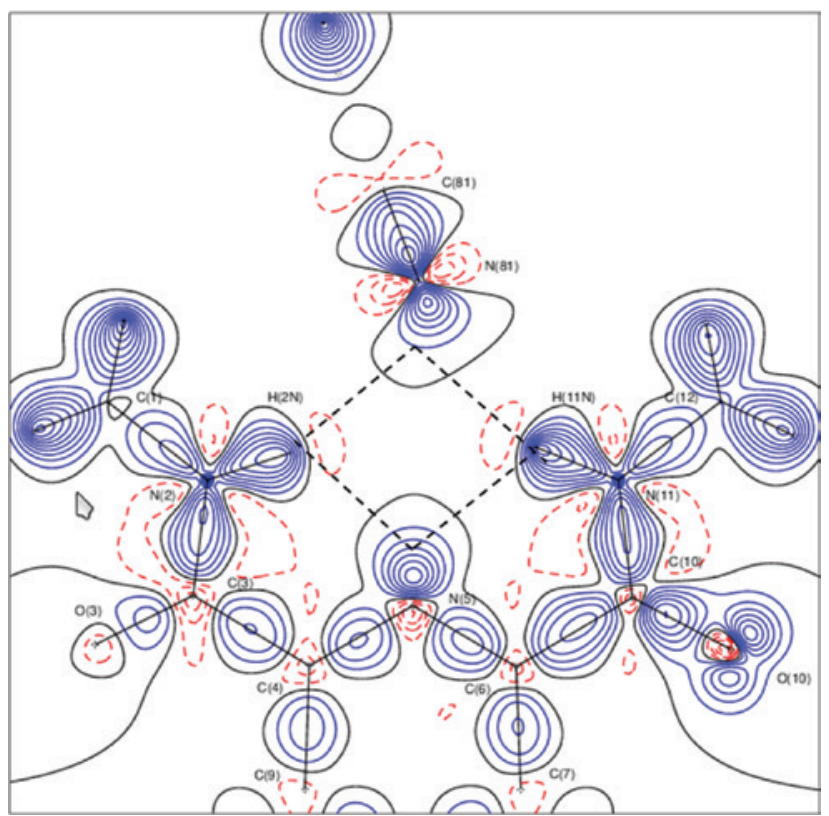

Fig. 6: Static deformation density map in the plane N(2), N(11), $\mathrm{N}(81)$ of $\mathbf{H}_{4} \mathbf{L}$ [14]; blue/black/red contour lines are for positive/ zero/negative densities; contour interval 0.1 e $\AA^{-3}$; hydrogen bonds in dashed lines. 
subprogram of XD [14] was used. Average results for the atomic charges and volumes are given in Table 2 only if the atomic charges are above $|0.2| e$.

All heteroatoms carry a strongly negative charge close to $-1 e$, which is in part compensated by the directly bonded carbon atoms; the strongest positive charge is at the amide carbon atom $(1.09 e)$, while the other named carbon atoms carry charges of 0.3-0.4 e. Carbon atoms not mentioned in Table 2 are practically neutral, which holds also for the hydrogen atoms except the ones being HB donors, having a significant positive charge of $\sim 0.4 e$ and a strongly reduced atomic volume of $3.7 \AA^{3}$. Hydrogen atom volumes are generally much larger, between 6 and $10 \AA^{3}$.

As requested by the keep charge option in the XD refinement each of the individual molecules are neutral for both $\mathbf{H}_{2} \mathbf{2}$ and $\mathbf{H}_{4} \mathbf{L}$, which holds also for the acetonitrile solvate molecules. For $\mathbf{H}_{2} \mathbf{2}$ the molecular volumes as obtained from the summation over the atomic volumes are $689.5 \AA^{3}$ for the macrocycle and $672.7 \AA^{3}$ for the thread (difference 2.5\%). The sum of both molecular volumes of $1362.2 \AA^{3}$ reproduces half the unit cell volume within $0.4 \%$, which is a good check whether the integration procedure has worked properly.

For $\mathbf{H}_{4} \mathbf{L} 7$ the molecular volumes of the two (chemically identical) macrocycles are 677.1 and $682.2 \AA^{3}$ (difference $0.8 \%)$. Together with the volumes of the two acetonitrile molecules (71.3 and $67.7 \AA^{3}$ ) the sum of all four molecular volumes of $1498.4 \AA^{3}$ is within $0.3 \%$ equal to half of the unit cell volume.

The electrostatic potential (ESP) surfaces shown in Figs. 7 and 8 were calculated by using the method of Volkov et al. [17] with the XDPROP subprogram of XD2006 [14] and color-coded onto the 0.067 e $\AA^{-3}(=0.01$ a.u.) ED isosurface using the graphical representation with MoLIso [13].

Table 2: Averaged atomic properties for atoms with an atomic charge $>|0.2| e$ (charges $q$ and volumes $V_{\text {tot }}$ ).

\begin{tabular}{lrrr}
\hline Atom type & $\boldsymbol{q}(\boldsymbol{e})$ & $\boldsymbol{V}_{\text {tot }}\left(\AA^{3}\right)$ & $\mathbf{N}^{\mathbf{a}}$ \\
\hline Oamid & $-0.90(2)$ & $19.0(17)$ & 6 \\
Oether & $-0.92(4)$ & $15.5(8)$ & 10 \\
Namid & $-0.93(1)$ & $13.2(7)$ & 6 \\
Npyridine & $-0.79(-)$ & $14.3(5)$ & 4 \\
Camid & $1.09(2)$ & $7.0(2)$ & 6 \\
C(-Namid) & $0.30(1)$ & $7.6(2)$ & 6 \\
Cpyridine(-N) & $0.32(1)$ & $9.3(5)$ & 8 \\
Csp $(-0)$ & $0.39(2)$ & $9.1(5)$ & 8 \\
Csp $3(-0)$ & $0.36(2)$ & $7.3(2)$ & 12 \\
Hhb $^{\text {b }}$ & $0.41(-)$ & $3.7(7)$ & 4 \\
\hline
\end{tabular}

${ }^{\mathrm{a}} \mathrm{N}$, number of entries contributing to the average; ${ }^{\mathrm{b}}$ hydrogenbonded atoms.
For each of the two catenanes the following types of ESP calculations were carried out:

1. Separate ESP calculations for the two contributing macrocycles (displayed in Fig. 7 for $\mathbf{H}_{\mathbf{2}} \mathbf{2}$ ).

2. Common ESP calculation for both macrocycles (displayed in Fig. 8)

A comparison of the ESP surfaces from the single calculations for $\mathbf{H}_{2} \mathbf{2}$ represented in Fig. 7 with the corresponding surfaces of the common calculation (Fig. 8, above) shows that - as expected - the features are very comparable (as far as they are visible in Fig. 8!), so that no significant mutual influence of the contributing interlocked molecules appears. The same holds for the corresponding ESPs in $\mathbf{H}_{4} \mathbf{L}$. For this catenane the case of the single ESP for one molecule must not further be considered (yet it is displayed in the Supporting Information, Fig. S2), because of the chemical identity with the macrocycle in $\mathbf{H}_{2} \mathbf{2}$.

Justified by the findings mentioned above and the much clearer visibility a discussion of some ESP details can preferably be restricted on the single ESP representations in Fig. 7.

The structural fragment amide-pyridine-amide, which exists in the macrocycle of $\mathbf{H}_{2} \mathbf{2}$ and in both macrocyclic molecules of $\mathbf{H}_{4} \mathbf{L}$, causes an extended horseshoe shaped negative potential in this region ranging in parts into the cavities of the molecules in question.

For $\mathbf{H}_{2} \mathbf{2}$ the cavity of the macrocycle is filled by the pyridine ring of the thread (Fig. 7 left) generating $\pi \cdots \pi$ interactions with two phenyl rings of the macrocycle, as mentioned before (see also Fig. 4). All three aromatic rings show negative potentials on their surfaces, so that in the cavity of the macrocycle negative ESP and hence repulsive interactions dominate.

The cavity of the thread (Fig. 7 right) is filled mainly by the phenyl ring $\mathrm{C}(31) \cdots \mathrm{C}(36)$ of the macrocycle. This phenyl ring is neighbored on one side to the pyridine ring of the thread in the sense of the already mentioned parallel displaced $\pi \cdots \pi$ interaction and on the other side to the aliphatic chain $C(57) \cdots C(64)$. There is a potential gradient in the thread cavity from the extended negative region of the arene/heteroarene pair to the positive potential of the aliphatic chain (see also Fig. 8, above).

The cavities of both $\mathbf{H}_{4} \mathbf{L} 7$ macrocycles are penetrated by the aliphatic chains $C(61) \cdots C(69)$ in case of molecules 1 and $C(20) \cdots C(29)$ in case of molecules 2 . In the interior of both cavities the negative potential caused by the above mentioned horseshoe type range of the fragment amidepyridine-amide interacts with the more positive potential of the aliphatic chains, so that an attractive ESP complementarity in the $\mathbf{H}_{4} \mathbf{L} 7$ cavities is established (see also Fig. 8, below). 
$\int_{-0.128}^{0.224 \mathrm{e} / \mathrm{A}}$
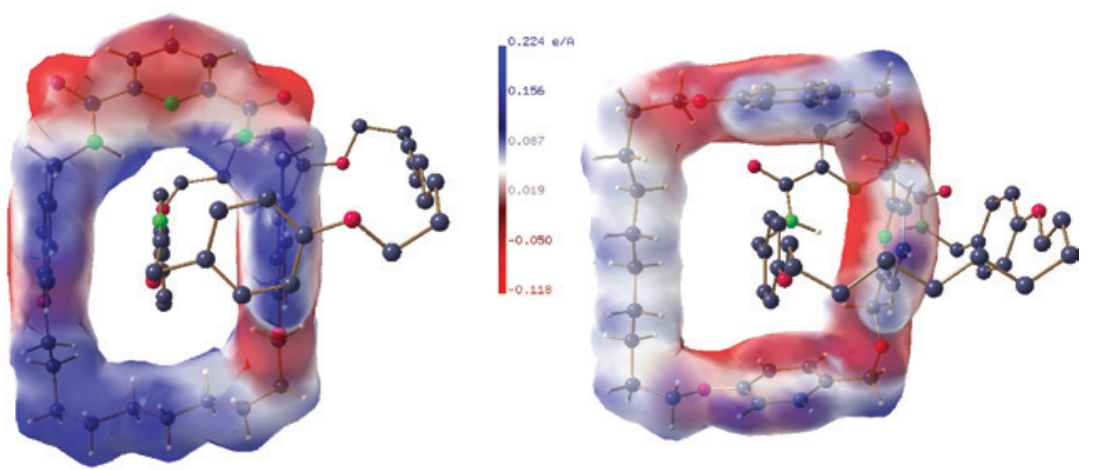

Fig. 7: Left: electrostatic potential of the macrocycle of $\mathbf{H}_{2} \mathbf{2}$; the thread is in a ball-and-stick style. Right: ESP of the thread; the macrocycle is in a ball-and-stick style; representations with MoLıso [13].
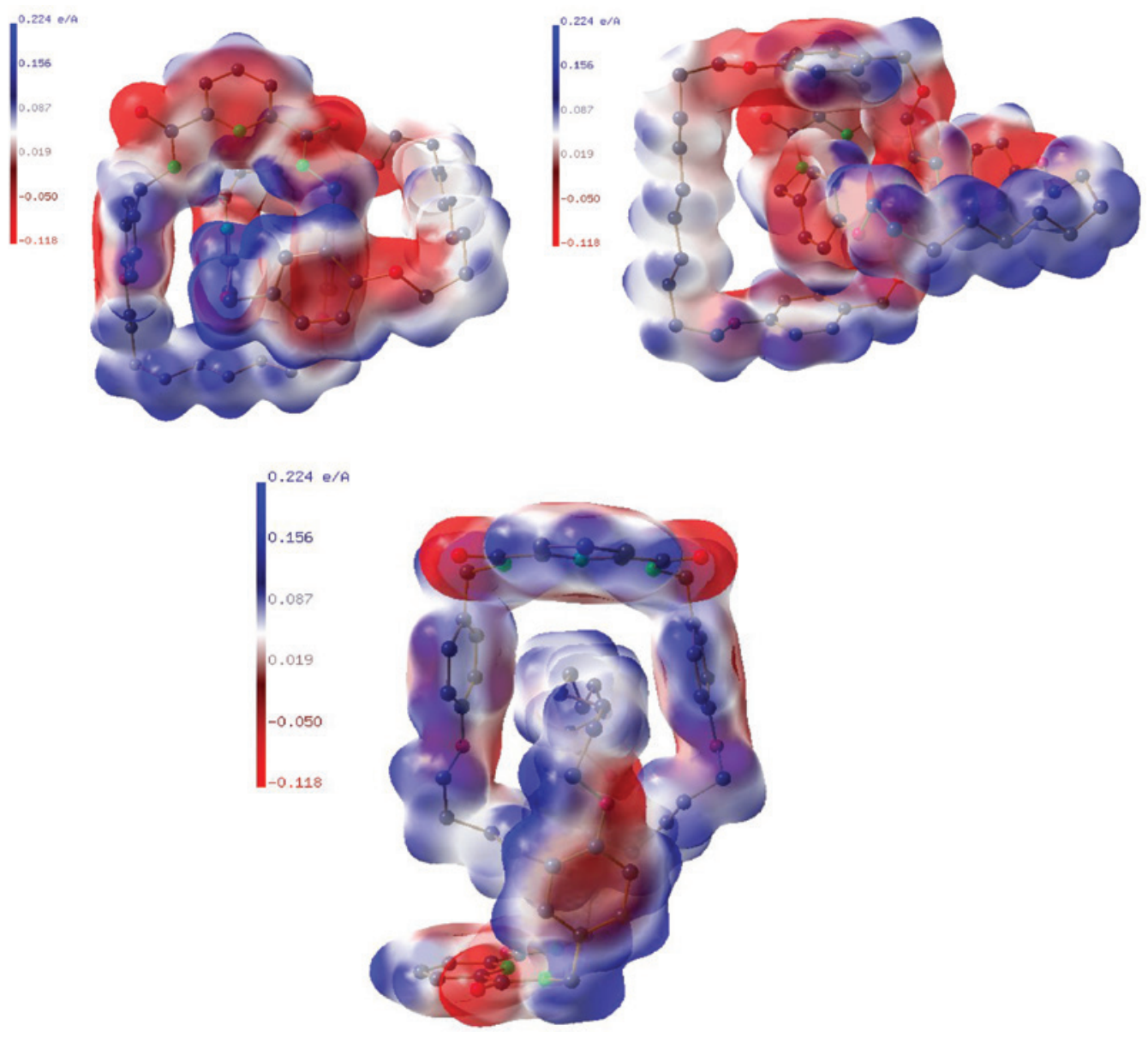

Fig. 8: Top left: representation of the common ESP of $\mathbf{H}_{2} \mathbf{2}$ in an orientation comparable to the one in Fig. 7 (left). Top right: Representation in an orientation comparable to the one in Fig. 7 (right). Bottom: Common ESP of both macrocycles of $\mathbf{H}_{4} \mathbf{L}$.

\subsection{Results from density functional theory}

The bonding analysis of the [2]catenanes based on experimental data is supported by density functional theory calculations at the B3LYP/TZVP [18-20] level applying Gaussian09 [21]. In the computations, the positions of the non-hydrogen atoms were kept fixed, whereas the hydrogen atom positions were optimized in the gas phase in order to properly render the weak intraand intermolecular interactions. The wave function files 
(wfn) were subsequently used to derive the theoretical AIM properties [15] with the AIM2000 [22] software and non-covalent interactions index (NCI) [23] surfaces with NCIplot [24]. Additionally, electron localizability indicator (ELI-D) $[25,26]$ surfaces were generated with DGRID [27] using the fchk files from GAUSSIAN09. NCI and ELI-D provide a complementary picture of chemical bonding as the former is used to exclusively depict non-covalent bonding aspects, whereas the latter is best suited to describe covalent bonding aspects. Within the NCI scheme the reduced electron density gradient, $s(\mathbf{r})=[1 / 2$ $\left.\left(3 \pi^{2}\right)^{1 / 3}\right]|\nabla \rho| / \rho^{4 / 3}$, is plotted at specific iso-surfaces (typically at $s=0.5$ ) resulting in the formation of NCI basins which account for all types of non-covalent interactions in a chemical system. Mapping the second eigenvalue of the electron density Hessian, $\lambda_{2}$, on the NCI surface facilitates further bond characterization according to: $\lambda_{2}<0$ : attractive, $\lambda_{2} \approx 0$ : Van-der-Waals-like, and $\lambda_{2}>0$ : steric/ repulsive. The dissection of the electron pair densities according to the ELI-D scheme leads to the formation of electron pair basins representing core, bonding, and lone-pair electrons. The almost complete spatial separation of NCI and ELI-D suggest (partial) spatial separation of non-covalent and covalent bonding aspects, a matter of recent debate [28-31].

Figure 9 displays the theoretical AIM distribution of $\mathbf{H}_{\mathbf{2}} \mathbf{2}$ together with the corresponding NCI iso-surface. The corresponding ELI-D results are shown in Fig. 10. Results for $\mathbf{H}_{4} \mathbf{L} \mathbf{7}$ are collected in the Supporting Information, including also the calculated electrostatic potentials (Figs. S3-S8). Numerous $\mathrm{N}-\mathrm{H} \cdots \mathrm{N}, \mathrm{C}-\mathrm{H} \cdots \mathrm{H}-\mathrm{C}, \mathrm{C}-\mathrm{H} \cdots \mathrm{C} \pi$, and $\mathrm{C} \pi \cdots \mathrm{C} \pi$ bond critical points are visible in the quite complex AIM distribution indicating the coexistence of hydrogen bonds, (weaker) dihydrogen bonds, and $\mathrm{H} \cdots \pi$ and $\pi \cdots \pi$ interactions. The AIM approach suggests all interactions to be rather localized forming BCPs between atoms. This approach is clearly valid for most types of chemical interactions including covalent, polar-covalent and strong hydrogen bonds but may be questioned for many kinds of weaker non-covalent interactions, which are non-directional by definition. One way to overcome this is to plot the ED distribution on proper surfaces, such as the Hirshfeld surface (as was shown in the previous section), or to use the NCI, see Fig. 9. Covalent interactions are not visible at the given iso-surface, whereas hydrogen bonds appear as localized bluish-colored NCI basins, indicating attractive forces. The much weaker dihydrogen bonds and $\pi \cdots \pi$ interactions form extended and flat green colored NCI basins between the molecules indicating weak van-der-Waals-like interactions to play a dominant role in this catenane type structure. Red colored NCI

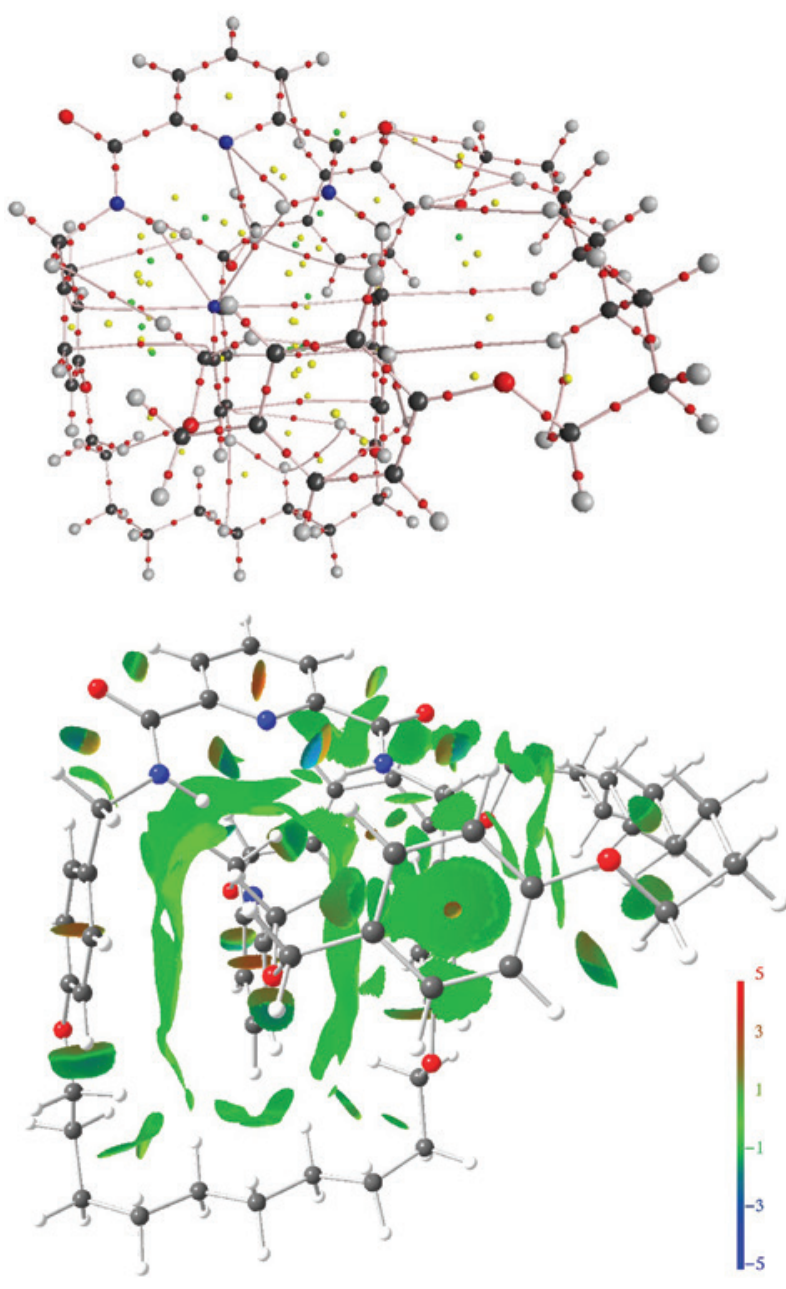

Fig. 9: AlM distribution (above) and $\mathrm{NCl}$ iso-surface at $s=0.5$ (below) of the calculated $\mathbf{H}_{2} \mathbf{2}$ structure. Bond critical points are given as red dots. AIM is plotted with AIM2000 [22], the $\mathrm{NCl}$ is plotted with MoLıso [13].

basins indicating steric clashes are not observed between both molecules but only in the center of the six-membered rings, as observed in earlier studies. As anticipated, the ELI-D iso-surface covers all covalent $\mathrm{C}-\mathrm{N} / \mathrm{O} / \mathrm{C} / \mathrm{H}$ and $\mathrm{N}-\mathrm{H}$ bonds as well as the lone-pair electrons of $\mathrm{N}$ and $\mathrm{O}$ atoms but leaves a void between both molecules in $\mathbf{H}_{2} \mathbf{2}$ (see Fig. 10), proving all intermolecular interactions to be of noncovalent type. ELI-D further provides an estimate of the spatial requirements of the ring molecules, which together with formation of weak interactions may influence the relative orientation of both molecules in the catenane structure. ELI-D basins covering $\mathrm{H}$ atoms are greenish colored (small) and flattened if they are located in the inter-ring area, which is a consequence of the many $\mathrm{H} \cdots \mathrm{H}$ contacts between the rings, whereas they are bluish colored (large) and form conic balloon shapes if they are located at the outer parts of the molecules, indicating the lack of 

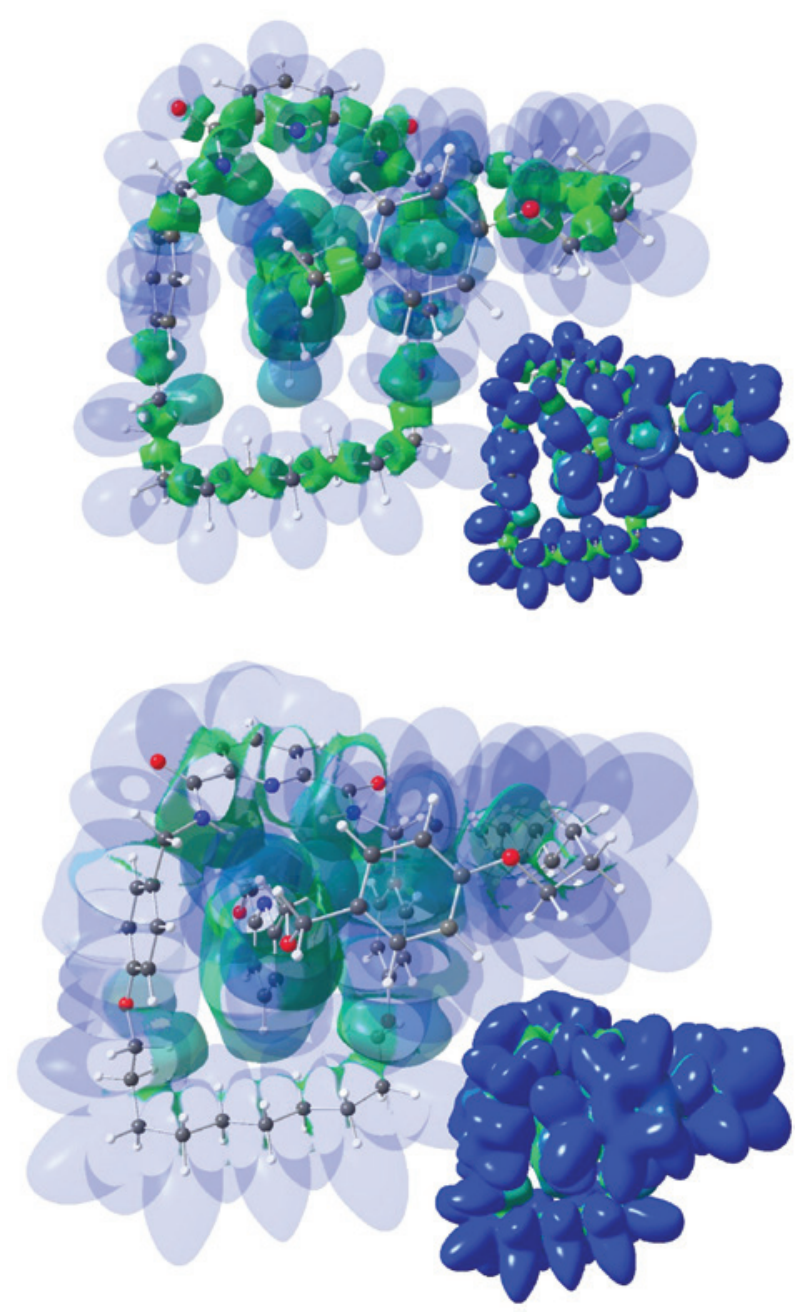

Fig. 10: ELI-D iso-surfaces, $s=1.2$ (above), and 0.6 (below) of the calculated $\mathrm{H}_{2} \mathbf{2}$ structure. Color code is roughly related to basin size going from greenish/solid for small basins to bluish/transparent for large basins. Inserts show the same surfaces in solid mode. The ELI-D representations are plotted with MoLISo [13].

any secondary chemical interactions. This is of course only true for the gas phase structures, since in the crystal structure $\mathrm{H} \cdots \mathrm{H}$ contacts appear also between different symmetry related catenane molecules. The ESP distributions confirm the NCI results. Polarized intermolecular interactions such as hydrogen bonds are characterized by a positive (blue-colored) ESP at the hydrogen bond donor site and negative (red-colored) ESP at the acceptor side, see Supporting Information (Figs. S2-S6). In contrast, for the non-polar dihydrogen bonds, blue regions meet blue regions and for the non-polar $\pi \cdots \pi$ interactions, red regions meet red regions. A comparison of the invariom derived ESPs with the corresponding theoretical ones shows a quite proper agreement. It seems that the polarization between positive and negative regions is more pronounced in the theoretical ESPs. There is a tendency of the ESP distributions to be somewhat more diffuse in the invariom derived ESPs compared to the theoretical ones and that the negative regions are less extended in the theoretical distributions, a property already seen in earlier studies [32, 33].

\section{Conclusion}

From the analysis of the invariom derived electron densities quantitative intra- and intermolecular bond and atomic properties of both [2]catenanes could be derived. In addition, molecular surfaces were calculated. Focus was directed to the identification and description of various types of intra- and intermolecular interactions. In addition to classical $\mathrm{N}-\mathrm{H} \cdots \mathrm{N}$ and $\mathrm{C}-\mathrm{H} \cdots \mathrm{O}$ hydrogen bonds, various types of $\pi \cdots \pi$ interactions exist. Most of them are verified by local ED concentrations visible on the corresponding Hirshfeld surfaces, except for the parallel $\pi \cdots \pi$ interactions in $\mathbf{H}_{\mathbf{2}} \mathbf{2}$, which are either too weak or too diffuse to generate an ED signal on the Hirshfeld surface between the contributing aromatic rings. The electrostatic potentials (ESPs) were calculated and illustrated on molecular iso electron density surfaces. The interactions in the cavity of one macrocycle with the penetrated fragment of the second one was examined. In the macrocyclic cavity of $\mathbf{H}_{2} \mathbf{2}$ three aromatic rings cause sandwich and parallel displaced type $\pi \cdots \pi$ contacts so that negative and hence repulsive interactions exist in this cavity. In the cavity of the thread repulsive negative ESP between the phenyl ring $\mathrm{C}(31) \cdots \mathrm{C}(36)$ of the macrocycle and the pyridine ring of the thread on one hand and attractive ESP with the aliphatic chain $C(57) \cdots C(64)$ exists on the other hand, so that in total a potential gradient is established. In $\mathbf{H}_{4} \mathbf{L} \mathbf{7}$ both macrocycles are chemically identical and the macrocyclic cavities are comparable. They are characterized by a positive potential of the aliphatic chain in its interior and the extended negative potential of the horseshoe type fragment amide-pyridine-amide. It follows that in contrast to $\mathbf{H}_{\mathbf{2}} \mathbf{2}$ only attractive interactions in the cavities are seen.

Non-covalent interactions from theoretical calculations in the gas phase were illustrated on NCI and ELI-D surfaces, where the spatial requirements of the macrocycles were displayed using ELI-D surfaces.

To summarize: Experimental Hirshfeld and ESP molecular surfaces and theoretical NCI and ELI-D surfaces are properly suited for an analysis of the structural and electronic interactions in the two examined [2]catenanes. 


\section{Invariom refinement}

For the invariom refinements of $\mathbf{H}_{2} \mathbf{2}$ and $\mathbf{H}_{4} \mathbf{L} \mathbf{7}$ the data sets reported in Ref. [6] and [7] were used. The known atomic parameters from the conventional spherical models were taken to establish the starting parameters for the subsequent aspherical-atom refinement. For aspherical modeling the software INVARION TOOL [34] was applied, which automatically selects and assigns the corresponding invarioms after analysing the identity and chemical neighborhood of each atom. Scattering factors up to the hexadecapolar level, including $\kappa$ parameters, from the invariom library were assigned to all atoms. Bond distances to hydrogen atoms were elongated to values from energy-minimized structures of the respective model compounds also used in scattering factor assignment. Hydrogen positions were idealized and riding model constraints were generated with the INVARION TOOL. After invariom transfer, refinement of positional and displacement parameters (anisotropic for non-hydrogen atoms, isotropic for hydrogen atoms) was carried out making use of the XD2006 program suite [14] until convergence was achieved. No charge transfer was allowed neither between the contributing macrocycles of each [2]catenane nor to the acetonitrile solvate molecules in $\mathbf{H}_{4} \mathbf{L} \mathbf{7}$. Selected crystallographic data and figures of merit are summarized in Table 3.

Table 3: Selected crystallographic and invariom refinement data for $\mathrm{H}_{2} \mathbf{2}$ and $\mathrm{H}_{4}$ L7. ${ }^{\mathrm{a}}$

\begin{tabular}{lll}
\hline Compound & $\mathrm{H}_{2} 2$ & $\mathrm{H}_{4} \mathrm{~L} 7$ \\
\hline Formula & $\left(\mathrm{C}_{31} \mathrm{H}_{37} \mathrm{~N}_{3} \mathrm{O}_{4}\right)\left(\mathrm{C}_{31} \mathrm{H}_{39} \mathrm{NO}_{4}\right)$ & $2\left(\mathrm{C}_{31} \mathrm{H}_{37} \mathrm{~N}_{3} \mathrm{O}_{4}\right) 2\left(\mathrm{C}_{2} \mathrm{H}_{3} \mathrm{~N}\right)$ \\
$M_{\mathrm{r}}$ & 1005.27 & 1113.38 \\
Crystal system & Triclinic & Triclinic \\
Space group (no.) & $P \overline{1}($ no. 2$)$ & $P \overline{1}($ no. $)$ \\
$Z$ & 2 & 2 \\
$V\left(\AA^{3}\right)$ & $2736.2(5)$ & $3005.8(8)$ \\
$T(\mathrm{~K})$ & $93(2)$ & $93(2)$ \\
$(\sin \theta / \lambda)_{\max }\left(\AA^{-1}\right)$ & 0.604 & 0.602 \\
No. of reflections & 15539 & 19224 \\
Observed refl. & 13232 & 16560 \\
{$\left[F_{\mathrm{o}}^{2} \geq 2 \sigma\left(F_{\mathrm{o}}^{2}\right)\right]$} & & \\
Invariom refinement & \\
$R(F)$ & 0.0463 & \\
$R_{\text {all }}(F)$ & 0.0568 & 0.0405 \\
$R_{\mathrm{w}}(F)$ & 0.0471 & 0.0528 \\
$R\left(F^{2}\right)$ & 0.0579 & 0.0407 \\
$R_{\text {all }}\left(F^{2}\right)$ & 0.0593 & 0.0538 \\
$R_{\mathrm{w}}\left(F^{2}\right)$ & 0.0921 & 0.0551 \\
$M i n /$ max $\Delta \rho\left(e \AA^{-3}\right)$ & $-0.40 / 0.78$ & 0.0808 \\
$\mathrm{GoF}$ & 2.517 & $-0.55 / 0.85$ \\
$N_{\text {ref }} / N_{v}$ & 17.8 & 2.012 \\
\hline & & 20.2 \\
\hline
\end{tabular}

aFor further data see CSD entries LASVIL and BOXCUN or Ref. [6] and [7].
In both refinements, the quantity $\Sigma w(\boldsymbol{h})$ $\left(\left|F_{0}(\boldsymbol{h})\right|^{2}-\left|F_{c}(\boldsymbol{h})^{2}\right|\right)^{2}$ was minimized by using the statistical weight $w(\boldsymbol{h})=1 /\left(\sigma^{2}\left(F_{0}(\boldsymbol{h})^{2}\right)\right.$ and only those structure factors, which met the criterion $F_{\mathrm{o}}^{2} \geq 2 \sigma\left(F_{\mathrm{o}}^{2}\right)$ were included.

\section{Supporting information}

Averaged bond properties for both [2]catenanes (Table S1) as well as further experimental and theoretical electrostatic potentials (Figs. S1-S8) are given as supplementary material available online (DOI: 10.1515/ znb-2018-0179).

Acknowledgement: The authors are grateful to the Deutsche Forschungsgemeinschaft (DFG) for financial support by project DI 921/6-1.

\section{References}

[1] G. Gil-Ramirez, D. A. Leigh, A. J. Stephens, Angew. Chem. Int. Ed. 2015, 54, 6110.

[2] Press Release of the Nobel Committee, October 2016, http:// www.nobelprize.org/nobel_prizes/chemistry/laureates/2016/ press.html. See also D. A. Leigh, Angew. Chem. Int. Ed. 2016, 55, 14506.

[3] F. H. Allen, Acta Crystallogr. 2002, B58, 380.

[4] B. Dittrich, T. Koritsanszky, P. Luger, Angew. Chem. Int. Ed. 2004, 43, 2718.

[5] B. Dittrich, C. B. Hübschle, K. Pröpper, F. Dietrich, T. Stolper, J. J. Holstein, Acta Crystallogr. 2013, B69, 91.

[6] D. A. Leigh, P. J. Lusby, A. M. Z. Slawin, D. B. Walker, Angew. Chem. Int. Ed. 2005, 44, 4557.

[7] D. A. Leigh, P. J. Lusby, R. T. McBurny, A. Morelli, A. M. Z. Slawin, A. R. Thomson, D. B. Walker, J. Am. Chem. Soc. 2009, $131,3762$.

[8] C. K. Johnson, ORTEP, Oak Ridge Thermal Ellipsoid Plot Program for Crystal Structure Illustrations, Rep. ORNL-6895, Oak Ridge National Laboratory, Oak Ridge, TN, USA, 1965.

[9] A. L. Spek, Acta Crystallogr. 2009, D65, 148.

[10] E. Keller, J. S. Pierrard, SCHAKAL99, Albert-Ludwigs University, Freiburg, Germany, 1999.

[11] J. J. McKinnon, A. S. Mitchell, M. A. Spackman, Chem. Eur. J. 1998, 4, 2136.

[12] M. A. Spackman, J. J. McKinnon, D. Jayatilaka, CrystEngComm. 2008, 10, 377.

[13] C. B. Hübschle, P. Luger, J. Appl. Crystallogr. 2006, 39, 901.

[14] A. Volkov, P. Macchi, L. J. Farrugia, C. Gatti, P. R. Mallinson, T. Richter, T. Koritsánszky, XD2006, A computer program for multipole refinement, QTAIM analysis of charge densities and evaluation of intermolecular energies from experimental and theoretical structure factors, University of Buffalo, NY (USA); University of Milano, (Italy); University of Glasgow, (UK); CNRISTM, Milano (Italy); Middle Tennessee State University, TN, USA, 2006. 
[15] R. F. W. Bader, Atoms in Molecules - A Quantum Theory, Clarendon Press, Oxford, 1994.

[16] A. Volkov, C. Gatti, Y. Abramov, P. Coppens, Acta Crystallogr. 2000, A56, 252.

[17] A. Volkov, H. F. King, P. Coppens, L. J. Farrugia, Acta Crystallogr. 2006, A62, 400.

[18] A. D. Becke, J. Chem. Phys. 1993, 98, 5648.

[19] C. Lee, W. Yang, R. G. Parr, Phys. Rev. 1988, B37, 785

[20] P. J. Stephens, F. J. Devlin, C. F. Chabalowski, M. J. Frisch, J. Phys. Chem. 1994, 98, 11623.

[21] M. J. Frisch, G. W. Trucks, H. B. Schlegel, G. E. Scuseria, M. A. Robb, J. R. Cheeseman, G. Scalmani, V. Barone, B. Mennucci, G. A. Petersson, H. Nakatsuji, M. Caricato, X. Li, H. P. Hratchian, A. F. Izmaylov, J. Bloino, G. Zheng, J. L. Sonnenberg, M. Hada, M. Ehara, K. Toyota, R. Fukuda, J. Hasegawa, M. Ishida, T. Nakajima, Y. Honda, O. Kitao, H. Nakai, T. Vreven, J. A. Montgomery, Jr., J. E. Peralta, F. Ogliaro, M. Bearpark, J. J. Heyd, E. Brothers, K. N. Kudin, V. N. Staroverov, R. Kobayashi, J. Normand, K. Raghavachari, A. Rendell, J. C. Burant, S. S. Iyengar, J. Tomasi, M. Cossi, N. Rega, J. M. Millam, M. Klene, J. E. Knox, J. B. Cross, V. Bakken, C. Adamo, J. Jaramillo, R. Gomperts, R. E. Stratmann, O. Yazyev, A. J. Austin, R. Cammi, C. Pomelli, J. W. Ochterski, R. L. Martin, K. Morokuma, V. G. Zakrzewski, G. A. Voth, P. Salvador, J. J. Dannenberg, S. Dapprich, A. D. Daniels, Ö. Farkas, J. B. Foresman, J. V. Ortiz, J. Cioslowski, D. J. Fox, GAussiAn 09 (revision B.01), Gaussian, Inc., Wallingford, CT, USA, 2010.
[22] F. Biegler-König, J. Schönbohm, D. A. Bayles, J. Comput. Chem. 2001, 22, 545.

[23] E. R. Johnson, S. Keinan, P. Mori-Sanchez, J. Contreras-García, A. J. Cohen, W. Yang, J. Am.Chem. Soc. 2010, 132, 6498.

[24] J. Contreras-García, E. Johnson, S. Keinan, R. Chaudret, J.-P. Piquemal, D. Beratan, W. Yang, J. Chem. Theor. Comp. 2011, $7,625$.

[25] M. Kohout, Int. J. Quantum Chem. 2004, 97, 651.

[26] M. Kohout; F. R. Wagner, Y. Grin, Theor. Chem. Acc. 2008, 119, 413.

[27] M. Kohout, DGRID-4.6, Radebeul, Germany, 2015.

[28] P. de Silva, C. Corminboeuf, J. Chem. Theory Comput. 2014, 10, 3745.

[29] N. Gillet, R. Chaudret, I. Contreras-García, W. Yang, B. Silvi, J.-P. Piquemal, J. Chem. Theor. Comput. 2012, 8, 3993.

[30] D. Fang, R. Chaudret, J.-P. Piquemal, G. A. Cisneros, J. Chem. Theor. Comput. 2013, 9, 2156.

[31] S. Mebs, Chem. Phys. Lett. 2016, 651, 172.

[32] T. Koritsanszky, R. Flaig, D. Zobel, H. G. Krane, W. Morgenroth, P. Luger, Science 1998, 279, 356.

[33] R. Flaig, T. Koritsanszky, R. Soyka, L. Häming, P. Luger, Angew. Chem. Int. Ed. 2001, 40, 355.

[34] C. B. Hübschle, P. Luger, B. Dittrich, J. Appl. Crystallogr. 2007, 40, 623.

Supplementary Material: The online version of this article offers supplementary material (https://doi.org/10.1515/znb-2018-0179). 